\title{
Genetic causal attributions for weight status and weight loss during a behavioral weight gain prevention intervention
}

\author{
Megan A. McVay, PhD ${ }^{1,2}$, Dori M. Steinberg, $\mathrm{PhD}^{3}$, Sandy Askew, MPH${ }^{3}$, Kimberly A. Kaphingst, ScD 4,5 \\ and Gary G. Bennett, PhD ${ }^{3,6}$
}

Purpose: Emerging evidence suggests that attributing one's weight to genetics may contribute to the adoption of obesogenic behaviors. We investigated whether weight-related genetic attributions were associated with weight change during a weight gain prevention intervention.

Methods: Participants $(n=185)$ were from a randomized clinical trial of a digital health weight gain prevention intervention for black women ages $25-44$ years with body mass index $25.0-34.9 \mathrm{~kg} /$ $\mathrm{m}^{2}$. Weight-related genetic attributions (weight status attribution and weight loss attributions) were measured at baseline and 12 months.

Results: Among intervention participants, high genetic attribution for weight loss was associated with greater weight loss at 12 months

\section{INTRODUCTION}

The causes of excess adiposity are multifactorial and include genetic, psychosocial, environmental, and physiological factors. The relative importance of these factors is unknown, leaving individuals to develop their own attributions for the causes of excess weight. Theoretical models suggest that individuals' causal attributions for weight may in turn exert an influence on their weight-related behaviors, and that causal attributions to genetics may be especially influential. For example, attribution theory posits that causal attributions have three dimensions: locus of control (internal versus external), stability (changeable versus not changeable), and controllability (controllable versus uncontrollable). ${ }^{1}$ Causal attributions that are perceived to be more internal, stable, and uncontrollable are associated with reduced engagement in goal-directed behavior. ${ }^{1}$ Because genetic causal attributions tend to be internal, stable, and uncontrollable, ${ }^{2,3}$ they may contribute to suboptimal weight management behaviors.

Several studies have found deleterious effects of genetic attributions on health behaviors. For example, genetic causal attributions for lung cancer, hypertension, colon cancer, and skin cancer were associated with engaging in a greater number of risky health behaviors, such as cigarette smoking, consuming a poor-quality diet, and not using sunscreen. ${ }^{4}$ Specifically with
( -2.7 vs. $0.5 \mathrm{~kg}$ ) and 18 months ( -3.0 vs. $0.9 \mathrm{~kg}$ ). Among usual-care participants, high genetic attribution for weight status was associated with greater 18 -month weight gain $(2.9$ vs. $0.3 \mathrm{~kg})$. The intervention reduced the likelihood of high genetic attribution for weight loss at 12 months $(P=0.05)$. Change in the likelihood of genetic attribution was not associated with weight change over 12 months.

Conclusion: Impact of genetic attributions on weight differs for those enrolled and not enrolled in an intervention. However, weight gain prevention intervention may reduce genetic attribution for weight loss.

Genet Med advance online publication 20 August 2015

Key Words: genetic determinism; obesity; prevention; weight loss

regard to weight-related attributions and behaviors, a few observational studies have looked at associations between genetic attributions and weight-related behaviors; one study did not find an association ${ }^{5}$ and another found a positive association. ${ }^{6}$ In a study using an experimental design, researchers found that participants who were provided a genetics-based explanation for obesity consumed more food in a lab-based eating episode than those provided a psychosocial explanation. ${ }^{7}$ On the whole, this existing literature suggests that greater genetic attributions for weight may contribute to lower likelihood to enact weight management behaviors, possibly contributing to less weight loss or to weight gain during a weight management intervention. However, the impact of genetic attributions on weight change during a weight management intervention has not yet been tested, to our knowledge.

In addition to a possible influence of genetic attributions on response to a weight management intervention, it is also possible that a weight management intervention could lead to changes in genetic causal attributions for weight. The Common Sense Model suggests that causal attributions influence perceptions of appropriateness of a particular treatment. ${ }^{8}$ Specifically, a "symmetry heuristic" has been described, such that causal attributions that are biological in nature (including genetic) are associated with inferences that biologically-based treatments

\footnotetext{
${ }^{1}$ Center for Health Services Research, Durham VA Medical Center, Durham, North Carolina, USA; ${ }^{2}$ Department of Psychiatry and Behavioral Sciences, Duke University Medical Center, Durham, North Carolina, USA; ${ }^{3}$ Duke Global Digital Health Science Center, Duke Global Health Institute, Duke University, Durham, North Carolina, USA; ${ }^{4}$ Department of Communication, The University of Utah, Salt Lake City, Utah, USA; ${ }^{5}$ Huntsman Cancer Institute, The University of Utah, Salt Lake City, Utah, USA; ${ }^{6}$ Department of Psychology and Neuroscience, Duke University, Durham, North Carolina, USA. Correspondence: Megan A. McVay (megan.mcvay@duke.edu)

Submitted 3 March 2015; accepted 25 June 2015; advance online publication 20 August 2015. doi:10.1038/gim.2015.109
} 
are appropriate, whereas nonbiological causal attributions are associated with belief in the appropriateness of behavioral interventions. ${ }^{4,89}$ Although the existing data on the symmetry heuristic have focused on the possible effect of causal attributions on treatment preference and choice, the reverse relationship can also be posited: that experience with particular treatments may alter causal attributions. However, this has not yet been examined in weight management.

In this study we sought to determine whether baseline genetic causal attributions for weight status and weight loss were associated with weight change among participants enrolled in a weight gain prevention intervention or usual care. We hypothesized that greater genetic attributions would be associated with weight gain or less weight loss. We also examined whether a weight gain prevention intervention affected genetic attributions for weight status and weight loss. We hypothesized that involvement in a behavioral intervention might reduce genetic attributions for weight status and weight loss. These aims were examined as part of secondary data analyses from the Shape Program, a randomized controlled trial comparing a 12-month weight gain prevention intervention to usual care among premenopausal black women attending community health center clinics. ${ }^{10}$ In that trial, the intervention successfully prevented weight gain at 12 months (mean difference: $-1.4 \mathrm{~kg}$; $95 \%$ confidence interval: -2.8 to -0.1 ) and at 18 months (mean difference: $-1.7 \mathrm{~kg}$; 95\% confidence interval: -3.3 to -0.2 ).

\section{MATERIALS AND METHODS}

\section{Participants}

The Shape Program trial is described in detail elsewhere. ${ }^{10,11}$ Participants were eligible if they were black women aged 2544 years, had a body mass index between 25 and $34.9 \mathrm{~m} / \mathrm{kg}^{2}$, and had the ability to read and write in English. Participants were excluded if they were pregnant or within 12 months postpartum, had a myocardial infarction or stroke in the previous 2 years, or had profound cognitive, developmental, or psychiatric disorders. Patients were recruited from six community health centers in central North Carolina operated by Piedmont Health. Participants were required to have had at least one visit to the community health center in the past 24 months. Participants who appeared eligible via medical record abstraction were recruited with invitational brochures followed by a screening call for eligibility. Potentially eligible patients then attended an in-person baseline study visit. A total of 194 participants were recruited and randomized (see Consort in primary outcomes manuscript, ref. 10). Patients were randomized via a computer program, with equal allocation (1:1) across treatment arms. Study design precluded blinding of patients or study staff. ${ }^{12}$ After randomization, three participants in usual care and six in the intervention became ineligible (because of pregnancy, relocating, or cancer diagnosis) and were not included in these analyses. This trial was registered with clinicaltrials.gov and has the clinical trial registry identifier NCT00938535. The Duke University Institutional Review Board and the Piedmont
Health Board of Advisors approved all study procedures. Informed consent was obtained from all subjects.

\section{Usual care}

In addition to the current standard of care delivered by their providers, usual-care participants received semiannual newsletters covering topics unrelated to weight, nutrition or exercise (e.g., finances, health, and beauty).

\section{Intervention}

The Shape intervention used the interactive obesity treatment approach, which aims to produce energy deficits through modification of routine obesogenic behaviors (e.g., no sugary drinks, no fast food, walk 10,000 steps per day). Participants were assigned three tailored behavior change goals at study onset, and these goals were updated every 2 months. Behavior change goals were selected by an algorithm based on participants' need for a specific behavior change, self-efficacy, readiness, and intended caloric deficit. Participants received weekly interactive voice response calls, during which they were asked to report their progress toward their goals and received tailored feedback (e.g., description of trends in progress, reinforcement, short skills training tips). Participants also received monthly counseling calls, lasting 20 minutes, with registered dietitians trained in motivational interviewing principles. These calls focused on the identification of barriers to behavior change, resolving ambivalence about behavior change, providing skills training, and goal setting. Calls were based on a script guided by a web-based application. Participants also received regular skills training materials such as tracking logs and were given free access to local YMCAs. Additional details on the Shape Program intervention are provided in Foley et al. ${ }^{11}$

\section{Measures}

Clinical measures. Weight and height were measured on calibrated wall-mounted stadiometer (Seca model 214, Seca, Chino, CA) and electronic scales (Seca model 876), respectively. Measures were taken by trained research assistants with participants wearing hospital gowns.

Genetic attributions. Attributions for the role of genes in weight status, diabetes, and heart disease were assessed using a measure that was used in a previous study. ${ }^{12}$ To assess attributions for weight status in the current study, participants were asked, "How much do you think a person's weight is caused by genes?" with the response options of "completely," "mostly," "somewhat," "a little," and "not at all." Similar questions were asked with regard to perceived causes of heart disease and diabetes. Participants who endorsed completely or mostly for the role of genes were characterized as having "high genetic attribution" for weight status, and those endorsing other response options were considered to have "low genetic attributions" for weight status.

An item was also developed for this study to assess participants' perceptions about the role of genes in their own weight 
loss. The item stated: "My genes play a role in whether or not I lose weight." Response options were "strongly agree," "somewhat agree," "neither agree nor disagree," "somewhat disagree," and "strongly disagree." Those who endorsed strongly agree and somewhat agree were considered to have high genetic attribution for weight loss, and those who endorsed the other options were considered to have low genetic attribution for weight loss. Responses were dichotomized for both genetic attribution variables to reflect a conceptual distinction between perceptions of high and low attributions for the role of genetics in weight. ${ }^{12}$

Other psychosocial and behavioral measures. Psychosocial variables were measured at baseline. Body image was measured with the 14-item Figure Rating Scale, a validated measure of body image that is designed to assess current and past body size as well as perceived attractiveness of body figure drawings. ${ }^{13}$ Eating characteristics were measured with the Three Factor Eating Questionnaire, which has three subscales: dietary restraint, uncontrolled eating (sometimes called disinhibited eating), and emotional eating. ${ }^{14}$ Physical activity self-efficacy was measured with five items assessing confidence in ability to exercise under various challenging circumstances. ${ }^{15}$ Moderate and vigorous physical activity was measured with a six-item scale derived from the Behavioral Risk Factor Surveillance System. ${ }^{16}$ Participants also were asked to report whether they had attempted weight loss in the past year (yes or no) and how much weight gain they expected in the next 2 years $(0-5,5-10$, or $>10 \mathrm{lb})$.

\section{Statistical analyses}

Analyses included 185 participants who remained eligible at 12 months. We initially conducted chi-squared or two-sided $t$-tests of means to compare those low and high in genetic attribution for weight and weight loss on the baseline psychosocial and behavioral variables of interest. We also conducted a Spearman rank correlation on uncollapsed genetic attribution variables to obtain an association between genetic attribution for weight status and weight loss, given the importance of these two variables. To test our first aim related to association between genetic attributions and weight change, we conducted a longitudinal mixed-effects model analysis with the dependent variable of weight and the independent variables of genetic attribution, time, and the interaction of time and genetic attribution. Separate analyses were conducted in the intervention and the usual-care arms. Baseline age, education, and clinic site were included in the model as covariates. We used a restricted maximum likelihood estimation approach and specified a random intercept and unstructured covariance matrix. Next, we conducted that same analysis but with genetic attribution for weight loss as the variable of interest.

To achieve our second aim of determining whether the treatment changed genetic attributions, we conducted a repeated measures analysis using generalized estimating equation models with a binary distribution, a logit link function, and an unstructured covariance matrix. The independent variables in these models were treatment arm (usual care or intervention), time (baseline and 12 months), and the interaction of time and treatment group. The dependent variable was an indicator of genetic attribution level (high versus low), with attributions for weight and weight loss tested separately. Intervention site was included in these models as a covariate. In post hoc analyses to further probe our findings, we modeled the association between the change in weight and genetic attribution for weight loss over time, adjusting for site, and using a similarly specified generalized estimating equation model. Because we were primarily interested in probing observations from the intervention session for this post hoc analysis, we present findings from only that group, although results were similar in the usual-care group and when results were pooled.

\section{RESULTS}

\section{Descriptive data}

Study participants had a mean age of 35.4 years ( $\mathrm{SD}=5.5$ years). Mean weight at baseline was $81.1 \mathrm{~kg}(\mathrm{SD}=8.8 \mathrm{~kg})$, and mean body mass index was $30.2 \mathrm{~kg} / \mathrm{m}^{2}\left(\mathrm{SD}=2.5 \mathrm{~kg} / \mathrm{m}^{2}\right)$. A majority of participants were employed (71.4\%), and $26.5 \%$ were married or living with a partner. Most participants had an education level of less than a college degree (79.7\%) and an annual income of less than $\$ 30,000$ (74.3\%). Metabolic syndrome criteria were met by $30.8 \%$ of participants. The correlation between genetic attribution for weight status and genetic attribution for weight loss was $\rho=0.26(P=0.0004)$.

\section{Associations of baseline genetic causal attributions with other baseline characteristics}

Across both study groups, high genetic causal attribution for weight status was associated with high genetic causal attribution for diabetes, heart disease, and weight loss (Table 1). It was also associated with higher self-reported uncontrolled eating. High genetic causal attribution for weight loss was associated with lower physical activity self-efficacy and higher self-reported uncontrolled eating (Table 2).

\section{Associations between baseline genetic attributions and weight change}

In the intervention arm, genetic causal attribution for weight status was not associated with weight change at any measurement time point (Table 3 ). However, high genetic causal attribution for weight loss was associated with greater weight loss at 12 and 18 months (Table 3 ).

In the usual-care arm, high genetic causal attribution for weight status was associated with greater weight gain at 18 months (Table 3). However, genetic causal attribution for weight loss was not associated with weight change.

To determine whether observed associations between baseline genetic attributions and weight change were independent of other related variables, we conducted sensitivity analyses in which we added to the model as covariates those variables that were significantly related to genetic attributions in the bivariate associations. Thus, we repeated the regression 
Table 1 Associations of genetic attributions for weight with other clinical and psychosocial variables at baseline

\begin{tabular}{|c|c|c|c|c|}
\hline & \multicolumn{2}{|c|}{ Genetic attribution for weight status } & $\begin{array}{c}\text { Test } \\
\text { value }^{\mathrm{b}}\end{array}$ & $P$ value \\
\hline Mean age, years (SD) & $35.2(5.4)$ & $35.9(5.7)$ & -0.80 & 0.42 \\
\hline Less than HS degree & $11(8.3)$ & $7(14.9)$ & & \\
\hline HS degree through some college & $30(22.6)$ & $14(29.8)$ & & \\
\hline Low & $77(57.5)$ & $14(28.6)$ & & \\
\hline High & $57(42.5)$ & $35(71.4)$ & & \\
\hline Attribution of heart disease to genes, $n(\%)$ & & & 28.13 & $<.0001$ \\
\hline Low & $91(68.4)$ & $12(25.5)$ & & \\
\hline High & $42(31.6)$ & $37(75.5)$ & & \\
\hline Mean BMI, kg/m² (SD) & $30.0(2.6)$ & $30.7(2.4)$ & -1.56 & 0.12 \\
\hline Attempted weight loss in past year, $n(\%)$ & & & 1.48 & 0.22 \\
\hline Yes & $99(74.4)$ & $32(65.3)$ & & \\
\hline No & $34(25.6)$ & $17(34.7)$ & & \\
\hline Expected weight gain in 2 years (lb), $n(\%)$ & & & 1.15 & 0.56 \\
\hline $0-5$ & $49(37.1)$ & $14(28.6)$ & & \\
\hline $5-10$ & $59(44.7)$ & $25(51.0)$ & & \\
\hline$>10$ & $24(18.2)$ & $10(20.4)$ & & \\
\hline Body image (current-ideal discrepancy), median (Q1, Q3) & $2.0(1.0,2.0)$ & $2.0(1.0,2.0)$ & $4,505.0^{c}$ & $0.88^{c}$ \\
\hline Total mean minimum, moderate, or vigorous activity per day (SD) & $150.4(285.4)$ & $235.3(657.9)$ & $-0.41^{\mathrm{d}, \mathrm{e}}$ & $0.68^{\mathrm{d}, \mathrm{e}}$ \\
\hline
\end{tabular}

${ }^{a}$ Frequencies may not sum to $n$ because of missing data. ${ }^{b}$ Test value is the chi-square value for discrete variables and the $t$-test value for continuous variables. ${ }^{c}$ From Wilcoxon rank-sum test. 'Test performed on log-transformed values. 'Satterthwaite test for unequal variances.

BMI, body mass index; HS, high school; TFEQ, Three-Factor Eating Questionnaire; Q, quintile.

analyses described above; in weight status models we adjusted for uncontrolled eating, and in weight loss models we adjusted for uncontrolled eating and physical activity self-efficacy. The addition of these covariates did not change the direction or significance of any of the relationships examined, nor were the covariates significant predictors of weight change in the model.

\section{Effects of intervention on genetic attributions over time}

For genetic attribution for weight loss, we observed a significant time-by-treatment group interaction, indicating differential change over time between treatment arms in genetic causal attributions for weight loss (chi-squared $=6.08 ; P=0.05$ ). In particular, we found that, at baseline, the intervention and usual-care groups did not differ on the likelihood of high or low genetic causal attributions for weight loss, whereas at 12 months the intervention group participants were more likely to have low genetic causal attribution for weight loss compared with usual-care participants (Table 4 and Figure 1). No significant effects were observed for the likelihood of genetic attribution for weight status.

In post hoc analyses we tested whether change in genetic causal attribution observed in the intervention group was associated with change in weight in that arm. We found that the odds of having low genetic causal attribution for weight loss did not change significantly with weight over time $(P=0.75)$. For every $5 \mathrm{~kg}$ of weight lost between baseline and 12 months, the odds of having a low genetic causal attribution for weight loss increased by $5 \%$ (odds ratio: $1.05 ; 95 \%$ confidence interval: $0.77-1.45)$.

\section{DISCUSSION}

Contrary to our hypothesis, greater weight loss was observed during a weight gain prevention intervention among participants who believed that genes play a greater role in weight loss compared with participants who attributed a lesser role to genes. By contrast, among participants who did not receive a 
Table 2 Associations of genetic attributions for weight loss with other clinical and psychosocial variables

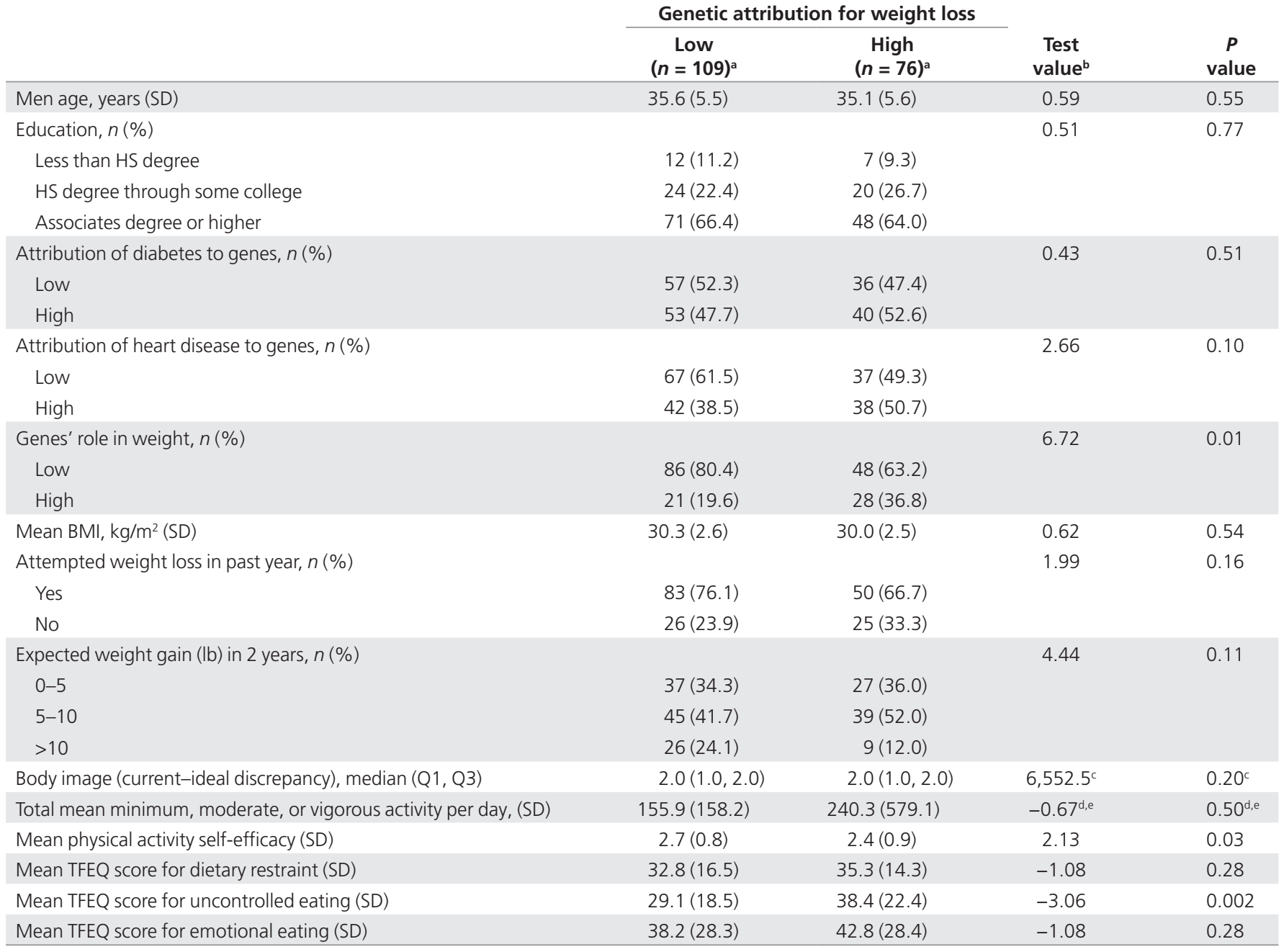

aFrequencies may not sum to $n$ because of missing data. ${ }^{\circ}$ Test value is the chi-square value for discrete variables and the $t$-test value for continuous variables. From Wilcoxon rank-sum test. ${ }^{\circledR}$ Test performed on log-transformed values. ${ }^{~}$ Satterthwaite test for unequal variances.

BMI, body mass index; HS, high school; TFEQ, Three-Factor Eating Questionnaire; Q, quintile.

Table 3 Relationship between baseline genetic attribution for weight status and weight loss, and weight change in intervention and control groups adjusting for site, baseline age, and education

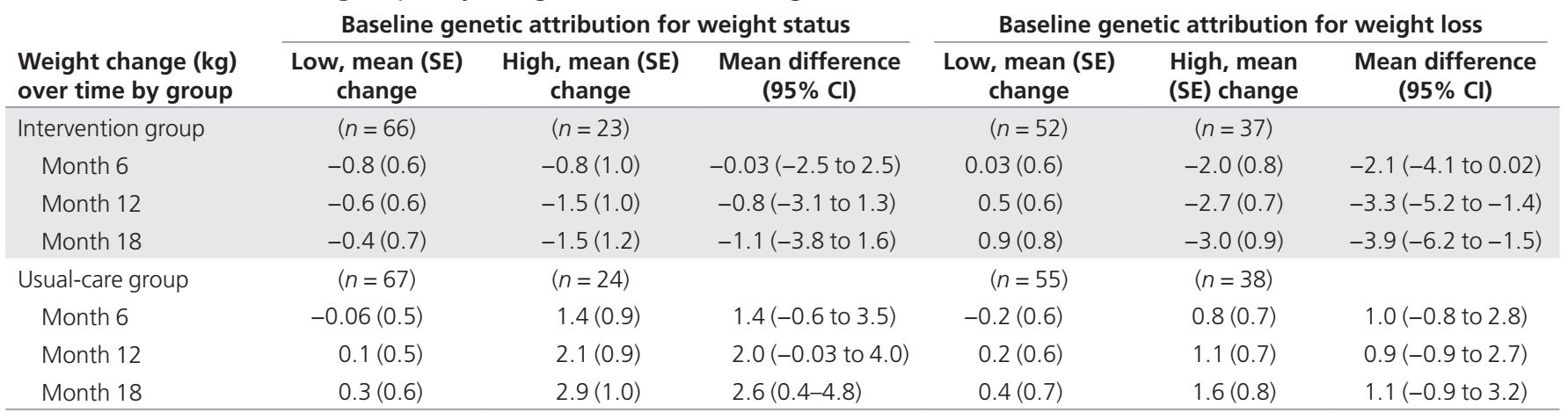

$\mathrm{Cl}$, confidence interval.

weight gain prevention intervention (usual-care arm participants), believing that genes play an important role in weight status was associated with weight gain over an 18-month period. We also found that participation in the weight gain prevention intervention led to a decrease in attribution of weight loss to genes over time. 
Table 4 Effects of weight gain prevention intervention on genetic attributions for weight and weight loss, adjusting for site

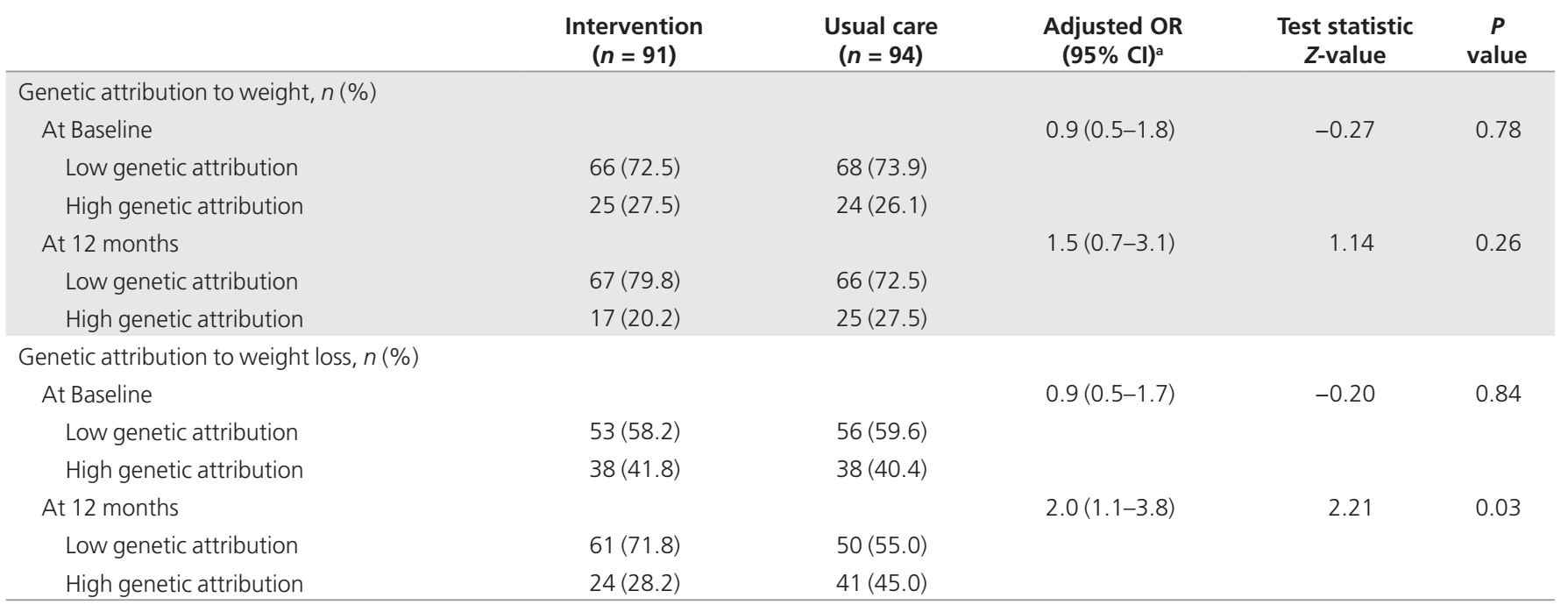

adds ratios (ORs) and $P$ values are for between-subjects comparisons at each time point. $\mathrm{Cl}$, confidence interval.

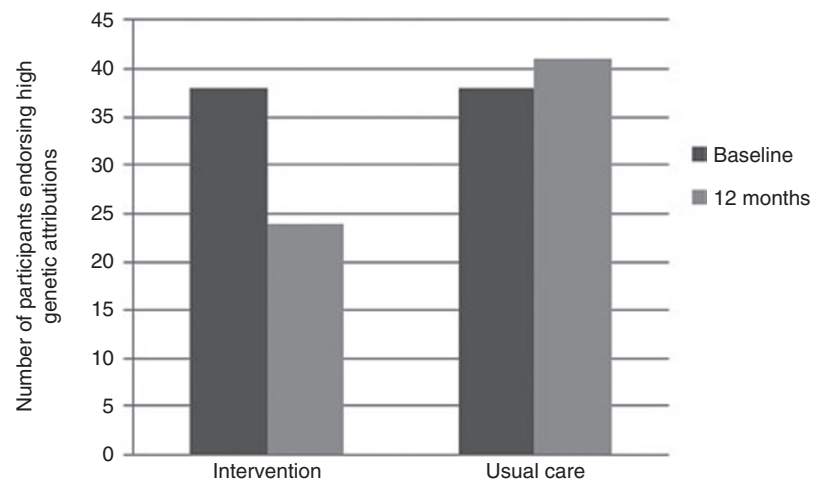

Figure 1 Endorsement of high genetic attribution for weight loss by study arm at baseline and 12 months.

Previous research has provided initial evidence that genetic causal attributions for weight may contribute to obesogenic behaviors. ${ }^{6,7}$ However, few studies have attempted to establish whether there is a relation between genetic causal attributions and outcomes in a behavioral weight management intervention. The current results suggest that higher genetic attributions for weight loss at baseline may contribute to greater weight loss for individuals enrolled in a weight gain prevention intervention. Notably, these effects were observed even after controlling for related concepts of uncontrolled eating and self-efficacy. One potential explanation we considered for this relation was that the intervention produced a reduction in genetic attributions, which in turn contributed to weight loss. Such an effect might be expected to have more benefit for those who start with higher genetic causal attributions, and thus could account for our observation. While this hypothesis was supported by our finding that the intervention reduced genetic causal attributions for weight loss, in further analyses we found that changes in attributions were not associated with weight change among the intervention participants.

This study is, to our knowledge, the first to show that participation in a weight management intervention can change perceptions about genetic causality for weight. This finding is consistent with past research highlighting the symmetrical relationship between causal attributions and treatment approach choice. ${ }^{8}$ To the extent that the current intervention increased participants' sense of personal control over their weight, it may have led to a concurrent reduction in beliefs of the role of genes. While this finding suggests that change in genetic attribution may be an active ingredient in the intervention, this is not supported by our follow-up analyses, which showed no association between change in genetic attribution and change in weight. Notably, this intervention had a weight gain prevention focus and achieved small weight losses; it is unclear whether results would differ in an intervention focused on weight loss where larger changes in weight would be expected. Further research is needed to confirm these findings and to extend them to weight loss interventions.

The observation that higher baseline genetic attributions were associated with greater weight loss during the intervention might suggest that it would be effective to discuss with weight management intervention participants the influence of genes in weight loss, while still emphasizing the importance of behavior change. However, the observed lack of association between change in genetic attributions and change in weight does not lend support to the value of intervening on this variable, and additional study is needed before recommendations are warranted. Future studies should test the effects of messages focused on the role of genes in weight loss during weight management interventions.

In contrast to intervention participants, among usual-care participants we observed that higher genetic causal attributions were associated with greater weight gain. In this study arm it 
was genetic causal attributions for weight status, not weight loss, that were significantly associated with weight change. These results indicate that, in the absence of a weight management intervention, genetic causal attributions about weight may indeed promote weight gain. Limited past research has examined the impact of genetic attributions on longitudinal weight outcomes in any population. A study by Hilbert et al. ${ }^{5}$ found that a measure of physiological/genetic attributions for obesity were not associated with weight change over a 6 -month period; however, this study was limited by the confounding of genetic and nongenetic physiological factors and the short follow-up period. Results of the current study are consistent with previous experimental ${ }^{7}$ and cross-sectional research ${ }^{6}$ linking genetic attributions to weight gain-promoting health behaviors. These results suggest that public health messages, and clinicians discussing weight with patients, should strive to focus on the importance of behavior change in weight control, especially for those who perceive genetic susceptibility.

In this study we separately considered genetic attributions for weight status and for weight loss. Analyses showed that, although these variables were significantly related, they seem to be distinct constructs with differential relationships to weight change. Further study of how these two types of weight-related genetic attribution are related to one another and to other variables is warranted. Qualitative evaluations in particular might provide additional information on differences in how individuals perceive the role of genetics in their current weight status compared with the role of genetics in weight loss. In addition, future studies focusing on genetic attributions related to weight may benefit from measuring these constructs separately.

At baseline, genetic attributions were associated with greater disinhibitions around eating. The reason for this association is unclear. It is possible that genetic attributions contribute to lower perceived control over eating. It is also possible that experiencing more difficulty controlling one's eating could contribute to the development of stronger genetic causal attributions. Additional longitudinal data may assist with clarifying the direction and causal nature of this relationship.

This study has several limitations. It is not known whether results will generalize to individuals who differ from our study participants, who were premenopausal black women in the primary-care setting. This study population is an important one, however, because black premenopausal women are at particularly high risk for weight gain. These findings also add to our understanding of causal attributions for weight status and weight loss in a study population with varying levels of educational attainment. Our analyses of the effect of genetic attributions on weight change are observational, and there may be unobserved confounding variables that are contributing to the association. We used single-item measures of causal attributions. In addition, this study focused specifically on perceptions about causality. A broader perspective that incorporates other constructs from theories that include causality (e.g., common sense model, attribution theory) may provide additional predictive value for weight change.
In summary, we found that high genetic causal attributions for weight loss at baseline were associated with greater weight loss during a weight gain prevention intervention. However, among participants not enrolled in an intervention (usual-care participants), high genetic attributions for weight status were associated with weight gain over an 18 -month period. We also found that participation in a weight gain prevention intervention contributed to a reduction in the likelihood of attributing weight loss to genes.

\section{ACKNOWLEDGMENTS}

This trial was funded by grant R01DK078798 from the National Institutes of Health. M.A.M. was supported by a postdoctoral training grant from the Veterans Affairs Office of Academic Affairs Health Services Research and Development (TPP 21-024). We are grateful to the administration and staff of Piedmont Health for their collaboration in the Shape Program and with the women who participated in Shape.

\section{DISCLOSURE}

D.M.S. has equity in Scale Down LLC. The other authors declare no conflict of interest.

\section{REFERENCES}

1. Weiner B. An attributional theory of achievement motivation and emotion. Psychol Rev 1985;92:548-573.

2. Jeong $\mathrm{SH}$. Effects of news about genetics and obesity on controllability attribution and helping behavior. Health Commun 2007;22:221-228.

3. Dar-Nimrod I, Heine SJ. Genetic essentialism: on the deceptive determinism of DNA. Psychol Bull 2011;137:800-818.

4. O'Neill SC, McBride CM, Alford SH, Kaphingst KA. Preferences for genetic and behavioral health information: the impact of risk factors and disease attributions. Ann Behav Med 2010;40:127-137.

5. Hilbert A, Dierk JM, Conradt M, et al. Causal attributions of obese men and women in genetic testing: implications of genetic/biological attributions. Psychol Health 2009;24:749-761.

6. Wang C, Coups E. Causal beliefs about obesity and associated health behaviors: Results from a population-based survey. Int J Behav Nutr Phys Act 2010;7:19.

7. Dar-Nimrod I, Cheung BY, Ruby MB, Heine SJ. Can merely learning about obesity genes affect eating behavior? Appetite 2014;81:269-276.

8. Marteau TM, Weinman J. Self-regulation and the behavioural response to DNA risk information: a theoretical analysis and framework for future research. Soc Sci Med 2006;62:1360-1368

9. Wright AJ, Sutton SR, Hankins M, Whitwell SC, Macfarlane A, Marteau TM. Why does genetic causal information alter perceived treatment effectiveness? An analogue study. Br J Health Psychol 2012;17:294-313.

10. Bennett GG, Foley $P$, Levine $E$, et al. Behavioral treatment for weight gain prevention among black women in primary care practice: a randomized clinical trial. JAMA Intern Med 2013;173:1770-1777.

11. Foley $P$, Levine $E$, Askew $S$, et al. Weight gain prevention among black women in the rural community health center setting: the Shape Program. BMC Public Health 2012;12:305.

12. Ashida S, Goodman M, Pandya C, et al. Age differences in genetic knowledge, health literacy and causal beliefs for health conditions. Public Health Genomics 2011;14:307-316.

13. Stunkard AJ, Sørensen T, Schulsinger F. Use of the Danish Adoption Register for the study of obesity and thinness. Res Publ Assoc Res Nerv Ment Dis 1983;60:115-120.

14. Stunkard AJ, Messick S. The three-factor eating questionnaire to measure dietary restraint, disinhibition and hunger. J Psychosom Res 1985;29:71-83.

15. Marcus BH, Selby VC, Niaura RS, Rossi JS. Self-efficacy and the stages of exercise behavior change. Res Q Exerc Sport 1992;63:60-66.

16. Centers for Disease Control and Prevention. Behavioral Risk Factor Surveillance System user's guide. Atlanta, GA, 1998. 